\title{
The Role of Oxytocin, Prolactin, and Estrogen in Male Sexual Functions
}

\author{
Ata Sedik Ibrahim Elsayed ${ }^{1}$, Azab Elsayed Azab ${ }^{2 *}$ and Rabia A. M. Yahya ${ }^{3}$ \\ ${ }^{1}$ Department of Basic Medical Sciences, Inaya Medical College, Riyadh, Saudi Arabia. \\ ${ }^{2}$ Department of Physiology, Faculty of Medicine, Sabratha University, Libya. \\ ${ }^{3}$ Department of Pharmacology, Faculty of Medicine, Sabratha University, Libya.
}

*Corresponding Author: Azab Elsayed Azab, Department of Physiology, Faculty of Medicine, Sabratha, University, Libya.

Received date: January 09, 2022; Accepted date: January 25, 2022; Published date: February 04,2022

Citation: Ata S.I. Elsayed, Azab E. Azab and Rabia A. M. Yahya. (2022). The Role of Oxytocin, Prolactin, and Estrogen in Male Sexual Functions. J Clinical Research and Reports, 10(3); DOI:10.31579/2690-1919/228

Copyright: (c) 2022, Azab Elsayed Azab. This is an open access article distributed under the Creative Commons Attribution License, which permits unrestricted use, distribution, and reproduction in any medium, provided the original work is properly cited.

\section{Abstract}

Background: The dysfunction in sexual ability has effects on the quality of life in men. Oxytocin plays a role in sexual and social behaviors. Hyperprolactemic males had erectile dysfunction. Endogenous estrogens in men, are not only important for health integrity but can additionally cause the promotion of many diseases.

Objectives: The current review aimed to high light on the role of oxytocin, prolactin, and estrogen in male sexual functions. Oxytocin neurons have been heavily implicated in mediating sexual behavior in both humans and animals. Oxytocin hormone regulates social behaviors such as mating, maternal/paternal care, and bonding. Oxytocin levels rise during mating in females and males in humans and animals and may mediate anxiolytic/calming effects of sexual activity and antidepressant effects. Oxytocin's role in regulating erection and ejaculation has been studied in mice and rats. Oxytocinergic projections from the paraventricular nucleus to the hippocampus, medulla oblongata, and spinal cord facilitate penile erection. Prolactin (PRL) serves a dual function both as a circulatory hormone and as a cytokine. PRL is known to be involved in the control of male copulatory behavior in humans, mice, rats, and other rodent models. It release during copulation in males and has a positive modulation for various aspects of testicular functions which hinting at the crucial role of prolactin in male reproduction. The lower concentration of prolactin was associated with reduced seminal vesicle volume and ejaculate in the infertile human male. Hypoprolactinemia has been associated with premature ejaculation and erectile dysfunction. Hyperprolactinemia can be caused by tumors, drugs, or idiopathic, leading to alterations in sexual behavior as loss of libido and erectile dysfunction. It is also reported that it can cause endocrine disturbances leading to abnormal levels of testosterone, FSH, and LH. Chronic hyperprolactinaemia suppresses copulatory behaviour in animal models and yields sexual dysfunction and other side effects in men. In men, testosterone acting via its action on androgen receptors may be dependent on the action of aromatase enzyme on of testosterone and converting it to estradiol (E2). Estrogens act through ER $\alpha$ and ER $\beta$ at the plasma membrane and in the nucleus to regulate functions of many organs in men. The role of estradiol action on libido is seen at various levels of regulation, starting with direct effects in the brain. Not only does estradiol modulate sexual behavior in the adult male, it also appears to organize the early brain to program sexual behavior. The exact role of estradiol in each area of male sexual function including libido, spermatogenesis, and erectile function is difficult to determine. A complex balance of testosterone, estradiol, aromatase, and estrogen receptors in brain, testes, and penis, confirmed the indispensable and highly regulated hormonal interaction of estrogen in the male.

Conclusion: It can be concluded that oxytocin hormone regulates social behaviors such as mating, maternal/paternal care, and bonding. PRL is involved in the control of male copulatory behavior in humans, rats, mice, and other rodent models. Hypoprolactinemia has been associated with reduced seminal vesicle volume, premature ejaculation, and erectile dysfunction. Hyperprolactinemia can be caused by tumors, drugs, or idiopathic, leading to alterations in sexual behavior as loss of libido and erectile dysfunction. Also, hyperprolactemic males had erectile dysfunction. Estradiol modulates sexual behavior in the adult male, and appears to organize the early brain to program sexual behavior.

Keywords: oxytocin; prolactin; hypoprolactinemia; hyperprolactinemia; estrogen; male sexual functions; libido; erectile dysfunction 


\section{Introduction}

The dysfunction in sexual ability has effects on the quality of life in men. At least about $20 \%$ of men experience some forms of dysfunction in their sexual forces during different periods of their life. The relation between decrease in sexual function and age is well documented, this correlated to weight gaining and consequent diabetes and cardiovascular diseases with their complications. Although treatments with phosphodiesterase inhibitors drugs as sildenafil or other drugs for improving erection and sexual functions, responsiveness to these drugs are varying, and medications for other forms of sexual dysfunction are limited [16].

Oxytocin plays a role in social and sexual behaviors, especially in erectile function [17]. Oxytocin hormone regulates social behaviors such as maternal/paternal care, bonding, and mating. This hormone levels rise during mating in males and females in humans and may mediate anxiolytic/calming effects of antidepressant effects and sexual activities. On the other hand, parturition is associated with a surged release of oxytocin from the supraoptic nucleus and paraventricular nucleus, resulting in rapid initiation of maternal behaviors such as depression, postpartum anxiety, and aggression against intruders, and positive bond with offspring. Melanin concentrating hormone (MCH) is a hypothalamic neuropeptide that shares in several physiological actions with oxytocin such as social recognition and stress, interaction, sexual behavior, regulation of emotion, and reproduction, and maternal care [18].

$88 \%$ of males who are hyperprolactemic had erectile dysfunction and the studies on effects of bromocriptine in hyperprolactinemic individuals, demonstrated that the prolactin secretion was lowered and spermatogenesis which was altered was restored to normal [20].

The sex steroidal hormones estradiol and testosterone, the first of which is derived from the latter one, play important functional and developmental roles in different organs in human females and males. These roles include actions in the reproductive and non-reproductive organs as brain, bone, cardiovascular system, and liver. The evidence now indicates that and androgens in women and endogenous estrogens in men, are not only important for health integrity but can additionally cause the promotion of many diseases [28].

\section{Objectives}

The current review aimed to high light on the role of oxytocin, prolactin, and estrogen in male sexual functions.

\section{Oxytocin}

The targeting potential impairments of medications in brain circuits that underlie sexual function have newly recently garnered interest. Selective inhibitors for serotonin reuptake, which leads to an increase in serotonin in extracellular fluids, have been used in the treatment of rapid ejaculation. The melanocortin system has a role in erection function, and many drugs which targeted this system has been tested for erectile dysfunction treatment. The agonists of dopamine, as apomorphine was explored as erectile dysfunction and sexual motivation treatment. Understanding of the neurocircuitry mechanisms of sexual function may lead to more effective treatments [27].

Many preclinical and clinical studies are supported the involvement of the melanocortin system in sexual actions and sexual abilities. Melanocortins hormones, such as $\alpha$-melanocyte-stimulating hormone $(\mathrm{MSH})$, adrenocorticotropic hormone $(\mathrm{ACTH}), \gamma \mathrm{MSH}, \beta \mathrm{MSH}$, are produced from the proopiomelanocortin (POMC) polypeptide, that bind to the MC1R, MC2R, MC3R, MC4R, and MC5R melanocortin receptors with different degrees of affinities. Since the MC3R and MC4R are the primary subtypes expressed by the brain, these two may mediate the central effects of melanocortins. MC4 receptors are G protein-coupled receptors that can couple with $\mathrm{Gi} / \mathrm{o}, \mathrm{Gs}$, and $\mathrm{Gq}$ receptors and trigger a series of downstream pathways. The studies suggested that melanocortin receptors, MC4R, which have a role in satiety mediating, may be mediate sexual abilities and sexual actions. The administration of $\alpha$-melanocytestimulating hormone, which is an agonist for MC3R and MC4R, have been found to lead to erection in mice and rats. At three to six months of age, MC4R null mice show decreased motivation to engage in sexual activity and take a longer time to reach ejaculation [21].

Male mice with insensitive POMC-producing neurons to insulin and leptin show reduced production in $\alpha \mathrm{MSH}$ and MC4Rs expression. These mice showed a decrease in sexual motivation which is indicated by a reduction in mounting behavior during the initial sexual encounter; this phenotype is evident at four to six months of age, concurrent with increases in their weights and a decrease in insulin sensitivity [8].

The proopiomelanocortin neurons project to nuclei that express Sim1, such as the paraventricular nucleus, supraoptic nucleus, and regions of the amygdala including the basolateral amygdala and medial amygdala. The study of Semple and Hill (2018) showed that genetically reexpressing MC4Rs only in Sim1 neurons of MC4RKO mice restores both intromission and ejaculation efficiency at age 6 months [26]. The receptors MC4Rs in Sim1 neurons play a role in regulating feeding behavior; the weights of these mice were duplicated compared to control at the testing time. Therefore, it remains to be determined whether MC4Rs on Sim1 neurons directly regulate sexual function. The obesity is a necessary dysfunction factor.

Oxytocin neurons are found in the paraventricular and supraoptic nuclei, downstream of the proopiomelanocortin neurons. Oxytocin neurons have been heavily implicated in mediating sexual behavior in both animals and humans. These neurons are postulated to mediate the effects of MC4 receptor agonists on female sexual functions and partner preference and Semple et al. (2019) hypothesized that oxytocin neurons may mediate MC4R driven sexual behavior in male mice [27]. They examined the ability of MC4Rs on oxytocin and Sim1 neurons in maintaining sexual function using mice, 2-month-old, without obesityrelated to age. Their findings underline the feasibility of identifying specific neuronal targets for male sexual dysfunction treatment [27].

When the melanocortin 4 receptor (MC4R) is knocked out globally, male mice display low sexual desire, obesity, and difficulties in copulation; however, it is unclear whether these phenotypes are interdependent. To elucidate the neuronal circuitry involved in sexual dysfunction in MC4R knockouts, Semple et al. (2019) in their study, reexpressed the MC4R in these mice exclusively on a subset of Sim1 neurons, namely oxytocin neurons (tbMC4Roxt mice) or Sim1 neurons (tbMC4RSim1 mice) [27]. The groups were compared to age-matched mice to observe the effects of obesity. In this experiment, young MC4R null mice had no deficits in erectile function or sexual motivation. However, MC4R null mice were found to have an increased latency to reach ejaculation compared to control mice, which was restored in both tbMC4RSim1 and tbMC4Roxt mice. These results indicate that signaling of melanocortin via the MC4R on oxytocin neurons is important for normal ejaculation independent of the male's metabolic health [27].

Oxytocin's role in regulating erection and ejaculation has been studied in mice and rats. Oxytocinergic projections from the paraventricular nucleus to the hippocampus, medulla oblongata, and spinal cord facilitate penile erection. The results of Clement and Giuliano (2016) study, revealed that MC4R signaling in oxytocin neurons permits ejaculation but argue against this pathway directly mediating erectile function [5]. Systemic and intracerebroventricular (ICV) administration of oxytocin has been found to facilitate ejaculatory function, while intravenous (IV), ICV, and intrathecal administration of an antagonist of oxytocin receptor (GSK557296) have been caused inhibition in 
ejaculation. Similar studies have found a decreased latency to ejaculate in rabbits and rats after administration of oxytocin. The effect of oxytocin receptor ligands on the ejaculatory response may be due to the modulation of dopamine serotonin neurotransmission [5]

Genetic deletions of the peptide (MCH-KO) or its receptors (MCHR1-KO) result in mothering deficits similar to those seen in oxytocin receptor KO (OXTR-KO) mice26. Both $\mathrm{MCH}$ and oxytocin lower the threshold for the initiation of maternal behavior but are not involved in its maintenance. Therefore, the oxytocin and $\mathrm{MCH}$ systems may interact to modulate maternal behavior. While only $4 \%$ of oxytocin neurons express MCHR1, OXTR mRNA are expressed in the majority of $\mathrm{MCH}$ neurons and only rarely in other neurons in the lateral hypothalamus (LH). Electrophysiological studies report that oxytocin interacts and selectively excites MCH neurons but not any other LH neurons [30].

The very selective action of oxytocin and the specific location of its receptors on $\mathrm{MCH}$ neurons suggests that these neurons may mediate or modulate some of the oxytocin actions on maternal behavior and emotion. The study of Sanathara et al. (2018) showed that the MCH and oxytocin systems interact directly, nonetheless, whether oxytocin regulates maternal- or mating-regulated mood through oxytocin-MCH signaling is unknown [24]. It is plausible to speculate that oxytocin may exert parts of its facilitating actions on mating and maternal responses through interacting with $\mathrm{MCH}$ neurons.

In the study of Phan et al. (2020), they uncovered the role of the oxytocin-MCH signaling pathway in mood regulation [18]. This study resulted in four novel findings. First, we identified a selective role for oxytocin-MCH signaling in mood regulation but not in maternal behavior. Second, they demonstrated that the effects of oxytocin receptors deletion from MCH neurons on mood are sex-dependent. Third, they showed that parenting experiences and mating shape basal mood and mood responses to oxytocin receptors deletion from $\mathrm{MCH}$ neurons. Finally, this study showed that parenting experience and OXTR deletion from $\mathrm{MCH}$ neurons cause remapping of brain Arc expressions, and that lower depressive behavior is associated with higher and lower Arc expression in the VTA and PVN respectively.

\section{Prolactin}

Prolactin is a polypeptide hormone, which is primarily secreted by the anterior lobe of the pituitary gland and formed in lactotropic cells, decidua, myometrium, breast, lymphocytes, leukocytes, and prostate. Prolactin action is varied between many functions as endocrine functions, growth, and development, metabolism, osmoregulation, immunomodulation neurobiological functions, and reproduction. It was believed to be an important factor in female reproductive functions, as the development of mammary gland, lactation, maintenance of corpus luteum, in addition to its role in maternal behavior [20]. Prolactin is probably best known for its role in lactation and maternal nurturing behavior; however, it is influential over approximately 300 biological functions across species leading it to be identified as the most versatile of all hormones. It was suggested that prolactin serves a dual function both as a circulatory hormone and as a cytokine [13]. Originally named after its role in lactation, prolactin is released in response to numerous external factors and physiological states. Although some, such as stress, are shared by both sexes, others are sexually dimorphic. The latter ones include the release of prolactin in response to nipple stimulation in lactating females, the prolactin circadian surges in naturally cycling females, and prolactin release during copulation in males. In females, prolactin is fundamental in organizing a series of behavioural and physiological programs for preparing individuals for motherhood as decreases female receptivity after fertilization, promotes food intake and maternal behavior $[4,7,12]$.

The receptors of prolactin are also present in male reproductive organs, but its role in males is still under investigation. Several human studies were conducted recently and have shown that prolactin has positive modulation for various aspects of testicular functions which hinting at the crucial role of prolactin in male reproduction [20]. Evidence from cross-species studies suggests that prolactin has independently evolved to promote paternal investment in three distinct lineages: mammals birds and fish. It may be very likely that prolactin has evolved to serve a similar function in the hominin lineage to the modern Homo sapiens. Similar to other species, human fathers and mothers show gender-specific parental behavior [13].

Prolactin release is controlled by many factors secreted from the Hypothalamus-Pituitary-Gonadal axis. The blood level prolactin is based depends on the stimulatory or inhibitory effect of these factors. Dopamine has antagonistic action on prolactin secretion, prolactin itself regulates its release of negative feedback effects through its action dopaminergic neurons in the hypothalamus and causes dopamine release which subsequently inhibits prolactin secretion through binding with D2 receptors on lactotroph cells in the anterior pituitary. Prolactin is believed to control the gonadal function by directly increasing the concentration of LH receptors on Leydig cells in the testes and indirectly by regulating the release of gonadotropins from the anterior pituitary lobe. These effects showed that prolactin acts at neuroendocrine levels and has an important role in male reproduction and sexual behavior [20].

The regulating role of prolactin on various testicular functions by modulating the LH and FSH and receptors on Leydig cells and Sertoli cells, respectively. It has also a role in the meiotic division of germ cells. The immunohistochemistry studies demonstrated the presence of prolactin in interstitial cells of Lydig, germ cells, and Sertoli cells, but it was unclear if prolactin was stored in the testes or produced from them. Another study carried out, observed the transcript and protein levels of prolactin in testis. Prolactin transcript was mainly found in spermatogonia, Leydig cells, and preleptotene and pachytene spermatocytes in both adult and prepubertal mice. Guillaumot and Benahmed (1999) showed the presence of prolactin transcript in the Sertoli cell isolated in vitro after FSH treatment [11]. It was also shown that testicular levels in the testis were lower as compared to the anterior pituitary levels. Using immunoblotting and immunofluorescence, protein levels were detected in testis as well as sperm [20].

In the past few years, many studies were investigated the relation between hyper- and hypo-prolactinemia and human male reproductive and sexual behavior. Hyperprolactinemia can be caused by tumors, drugs, or idiopathic, leading to alterations in sexual behavior as loss of libido and erectile dysfunction. It is also reported that it can cause endocrine disturbances leading to abnormal levels of testosterone, FSH, and $\mathrm{LH}$. The study reviewed in Raut et al. (2019) review article, showed high levels of prolactin in patients with different infertile conditions like asthenozoospermia, oligozoospermia, and azoospermia. However upon treatment with bromocriptine, as prolactin antagonist, the serum prolactin concentration remained unaltered. These suggest that there are no sufficient shreds of evidence to prove if hyperprolactinemia leads to erectile dysfunction. The role of bromocriptine as an antagonist for prolactin action is well established and is used in erectile dysfunction treatment.

Colao et al. (1996) studied the effect of quanagolide as a dopamine agonist, in patients with microprolactinoma and macroprolactinoma and which showed lower testosterone, high levels of prolactin, along low sperm motility, and sperm count [6]. However, after treatment with quanagolide, the prolactin concentrations were lowered and the other parameters were returned to the normal levels. The same results were shown by treatment with cabergoline which acts as a dopamine agonist On the other extreme, very few studies reviewed by Raut et al. (2019) reported that individuals with sexual dysfunction showed a lower levels of prolactin and a higher risk of loss of libido and premature ejaculation 
[20]. Low prolactin levels may be caused an altered serotonin pathway or an increase in dopamine levels. The studies in this review find a strong relationship between hypoprolactinemia and a decrease in sperm motility, however, no effects were observed on other sperm parameters.

The lower concentration of prolactin was associated with reduced seminal vesicle volume and ejaculate in the infertile human male. In addition, hypoprolactinemia has been associated with premature ejaculation and erectile dysfunction in men consulting for sexual dysfunction. Recapitulating metabolic syndrome, and several metabolic derangements have also been associated with low levels of prolactin both from the general European population and in men with sexual dysfunction. The low levels of prolactin had been identified as an independent predictor of the incidence of major adverse cardiovascular diseases in men with sexual dysfunction. Finally, an association with anxiety or depressive symptoms has been found in men suffered from sexual dysfunction. While a direct role for impaired prolactin function in the pathogenesis of these psychological, metabolic, reproductive and sexual disorders is conceivable, the possibility that low prolactin is a mirror of decreased serotonergic tone or increased dopaminergic or a cannot be ruled out. Hyperactivity of the dopaminergic system can explain only a few of the aforementioned findings, whereas a hyposerotonergic tone fits well with the clinical features associated with low prolactin levels, and there is significant evidence supporting the hypothesis that prolactin could be a mirror of serotonin in the brain [19].

The key role of prolactin in maternal physiology has traditionally biased neuroendocrinological studies towards its functions in the brain of females, whereas the actions of this hormone in the male brain have drawn less attention. For instance, the distribution of the PRL receptor (PRLR) has been reported for females (Bakowska, and Morrell, 1997, 2003, Salais-Lo'pez et al., 2018) and only recently studied in the brain of male rodents $[1,2,15,23]$. Using a genetically modified animal in which a reporter ( $\tau \mathrm{GFP}$ ) is expressed under the control of the PRLR promoter, the authors of this study have shown that the expression of PRLR is similar in males and females with quantitative differences in only two nuclei, the anteroventral periventricular nucleus (AVPe) and the medial preoptic nucleus (MPO).

According to the findings of Salais-Lo'pez et al. (2018) study, it is suggested that prolactin may have many equivalent functions in both sexes [23]. One of the main reasons for the aforementioned bias in functional studies of prolactin relates to the fact that male rodent models display low prolactin circulating levels under standard conditions, reflecting limited access, and functionality of prolactin in the brain. Still, several studies do report acute rises in systemic prolactin secretion in males associated with certain physiological conditions, such as sexual behavior or stress response. Regarding the latter, PRL is known to be involved in the control of male copulatory behavior in humans, mice, rats, and other rodent models. Thus, prolactin is released acutely with ejaculation (Guillou et al., 2015, Bronson et al., 2017, Kirk et al., 2017), and it is proposed to intervene in the satiation following copulation leading to the refractory period $[3,12,14]$. Chronic hyperprolactinaemia suppresses copulatory behaviour in animal models and yields sexual dysfunction and other side effects in men. The inhibitory role of prolactin on sexual function is proposed to have an important central component, but the exact regions and mechanisms through which PRL operates in the male brain are still poorly understood [23].

\section{Estrogens}

The accumulated shreds of evidence reported that some of the biological actions in men, traditionally attributed to testosterone acting via its action on androgen receptors may be dependent on the action of aromatase enzyme on of testosterone and converting it to estradiol (E2). The circulating level, E2 in men is exceeding the levels in postmenopausal women, and estrogen receptors are expressed in many somatic and reproductive tissues of males. The collective studies suggested that, in men, estradiol is an important factor in hypothalamic-pituitary-testicular axis regulation, growth hormone insulin-like growth factor-1 axis regulation, reproductive function, maintenance of skeletal health and bone growth, body composition, and glucose metabolism and vasomotor stability. Many clinical studies support the use of testosterone as the treatment of choice in male hypogonadism, rather than aromatase inhibitors, selective androgen receptor modulators, and selective estrogen receptor modulators [22].

\subsection{Estrogen receptors in men}

The estrogen receptors ER $\alpha$ and ER $\beta$, which are encoded by genes ESR1 and ESR2 respectively, are nuclear receptors, there also are Gprotein-coupled estrogen receptor 1 (GPER) on the cell membrane. Estrogen receptors are expressed on the human male reproductive tract, and also on the cardiovascular system, bone, adipose tissue, pancreatic islets, skeletal muscle, liver, and in the male brain. Estrogen signaling occurs by ligand-dependent pathway, involves ligand-activated dimerized estrogen with direct DNA binding to estrogen response elements in gene promoters causing regulation of RNA transcription. $\mathrm{ER} \alpha$ and $\mathrm{ER} \beta$ bind to the same estrogen response elements. The estrogen receptors can also be activated to modulate gene transcription in a ligand-independent mode via second messenger kinases involved in signal transduction by receptors of growth factors. There are also non-genomic pathways by which ER ligand binding induces rapid physiological effects [22]. 

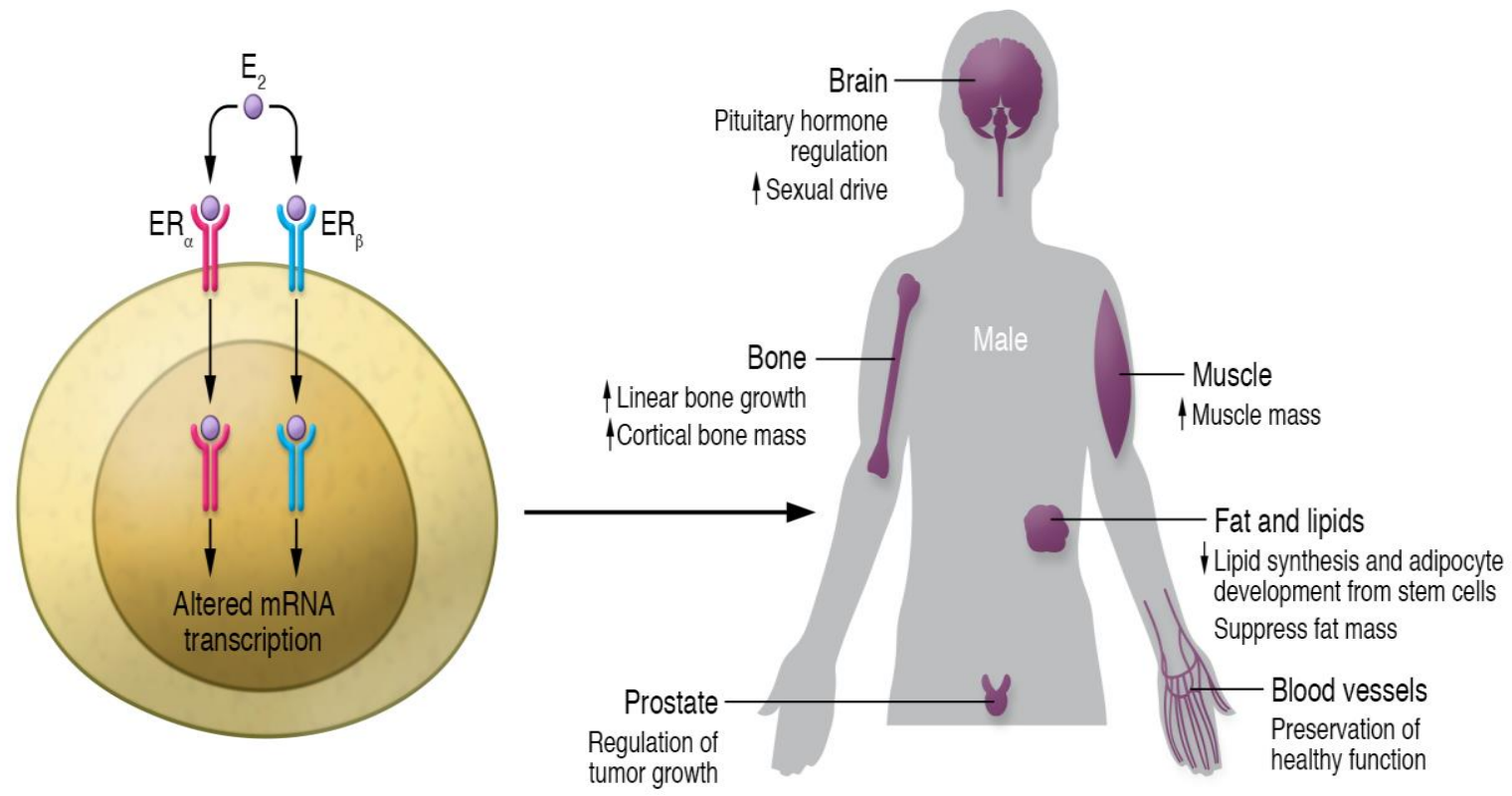

Figure 1: Estrogens act through ERQ and ERß at the plasma membrane and in the nucleus to regulate functions of many organs in men [28].

\subsection{Spermatogenesis}

Estrogen receptors ER $\alpha$ and ER $\beta$ are expressed throughout the human male reproductive tract, including in germ cells. The aromatase enzyme is found in human efferent ductules, epididymis immature germ cells, and spermatozoa. The experimental studies on mice demonstrated that estrogen has an important role in the signaling of male fertility, but the studies in human males have been less dramatic and less conclusive. The reviewed studies by Russell and Grossmann (2019) reported that male ER $\alpha$-knockout mice showed infertility due to disruption of fluid reabsorption in the epididymal efferent ductules [22]. Knockout mice exhibit progressively impaired spermatogenesis with aging. The reported results on testicular function in two men with homozygous missense mutations in ESRl causing non-functional ER $\alpha$ receptors. The first man, 28 year old, had descended testes of normal volume and normal sperm count with a minor reduction in sperm viability, and the second human male, 18 years old, had a cryptorchid right testis, hypoplastic left testis, and primary hypogonadism. The abnormalities have been reported in men with congenital aromatase deficiency. In most cases, testicular size was normal, although a few men had low testicular volume, and cryptorchidism has been commonly reported in these men. Where reported, sperm counts were normal or reduced with abnormal sperm motility. Studies on a few men with a deficiency in aromatase, the testicular biopsy showed varying degrees of spermatogenesis impairment and germ cell arrest. These observations suggest a role for E2 gene in spermatogenesis and testicular descent. The fact that some men appear to have normal testicular function despite congenital lack of E2 gene may be due to dietary supplementation with phytoestrogen, or the other explanation is that men with aromatase deficiency would be expected to retain ligand-independent $\mathrm{ER} \alpha$ signaling in the reproductive tract [22].

\subsection{Association between libido and estradiol}

\subsubsection{Role of estradiol in the brain}

The role of estradiol action on libido is seen at various levels of regulation, starting with direct effects in the brain. Areas of the brain that control sexual behavior in mammals are thought to do so via pheromones which induce specific sexual effects on the autonomic nervous system, including sexual arousal and mood changes. The action of pheromones increases medial preoptic area and anterior hypothalamus activities. Neurons and astrocytes glial cells in CNS convert testosterone to estrogen with the aromatase enzyme. The preoptic area and anterior hypothalamus contain the highest levels of the aromatase and estrogen receptors in male rodents. Similarly, it is well known that selective serotonin reuptake inhibitors diminish libido. Serotonin receptors follow a pattern of distribution similar to that of estrogen receptors in the brain. The activity of aromatase enzyme is highest in the brain during development. Thus, not only does estradiol modulate sexual behavior in the adult male, it also appears to organize the early brain to program sexual behavior [25]. 


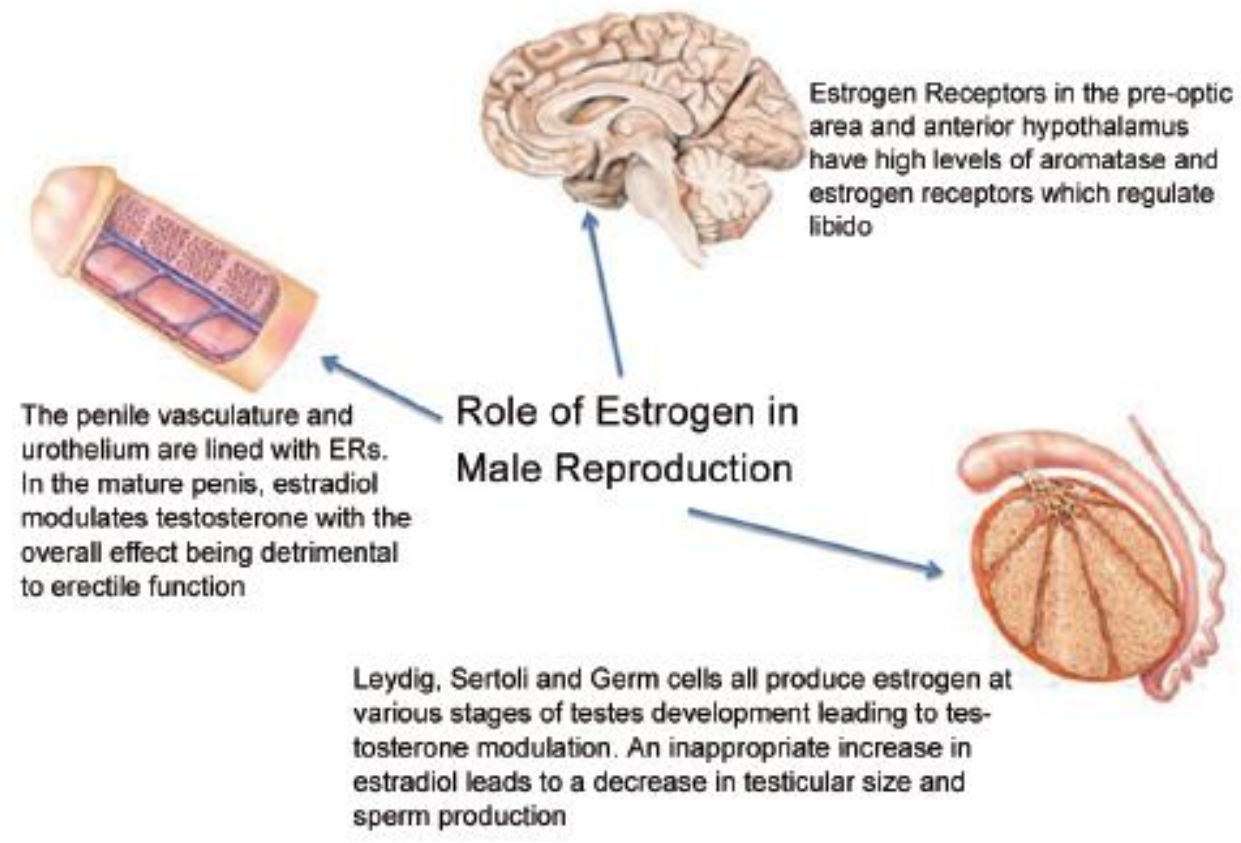

Figure 2: The role of estrogen in male reproduction [25]

The exact role of estradiol in each area of male sexual function including libido, spermatogenesis, and erectile function is difficult to determine. A complex balance of testosterone, estradiol, aromatase, and estrogen receptors in brain, testes, and penis, confirmed the indispensable and highly regulated hormonal interaction of estrogen in the male. Estrogen receptors and aromatase enzyme share topographic locations with pheromones in the brain, making it clear that estrogen contributes to sexual behavior and early sexual development in males during adulthood. Estrogen can sustain libido as well as affect the number of serotonin receptors in the brain modulating mood, cognition, emotion, and mental state. Erectile function is adversely affected by estrogen exposure in early penile development, and exposure to estradiol in the mature penis leads to increased vascular permeability with increased erectile dysfunction. Erectile dysfunction from increased estradiol exposure is independent of levels of testosterone. Spermatogenesis is also dependent on estradiol to some extent, as all cells involved in the process of spermatogenesis contain aromatase enzyme and express receptors of estrogen. Finally, estradiol levels should be considered when treating men with testosterone, as estradiol levels below $5 \mathrm{ng} / \mathrm{dl}$ correlate to a decrease in libido. More understanding of these mechanisms may prove the using of estradiol and become clinically useful in treating diminished libido, erectile dysfunction, and perhaps even oligospermia [25].

The evidence from men with congenital non-functional ER $\alpha$ or aromatase deficiency suggests that E2 is not essential for male libido and erectile function, but various lines of evidence support some physiological role. In non-blinded studies, two men with aromatase deficiency had increments in libido and sexual activity during E2 treatment, either alone or with testosterone. Non-definitive evidence from men with prostate cancer suggests that androgen deprivation therapy using estrogens may better maintain sexual function than surgical castration and GnRH analogs [10,29].

In the experiment of Finkelstein et al. (2013) they gave a GnRH analog with or without AI to 400 adult healthy men for sixteen weeks [9]. the volunteers were further randomized to add-back with one of five testosterone gel doses with transdermal, that produced serum levels of testosterone ranging from castrating to mildly supraphysiologic, either with E2 clamped to very low concentrations (AI cohort) or with the expected corresponding E2 increments for each testosterone dose increment (non-AI cohort). This experiment allowed for discrimination of testosterone from E2-dependent effects. Testosterone-related effects were assessed directly by comparing changes between groups of men in whom E2 was clamped. E2-related effects were inferred indirectly, by comparing mean change for each outcome in the AI cohort with the outcome in the non-AI cohort, and also by linear regression modeling of the cohort-testosterone dose interaction. In this analysis, E2 clearly showed a positive effect on erectile function and sexual interest, independent of testosterone. Assignment to the AI cohort was not randomized and there was no placebo, so the possibility of systematic differences between cohorts that were not due to differences in serum E2 is not completely excluded. It cannot be excluded that potential adverse effects of AI in addition to their E2-lowering actions, might have contributed to libido reductions.

\section{Conclusion}

It can be concluded that oxytocin hormone regulates social behaviors such as mating, maternal/paternal care, and bonding. PRL is involved in the control of male copulatory behavior in humans, rats, mice, and other rodent models. Hypoprolactinemia has been associated with reduced seminal vesicle volume, premature ejaculation, and erectile dysfunction. Hyperprolactinemia can be caused by tumors, drugs, or idiopathic, leading to alterations in sexual behavior as loss of libido and erectile dysfunction. Also, hyperprolactemic males had erectile dysfunction. Estradiol modulates sexual behavior in the adult male, and appears to organize the early brain to program sexual behavior.

\section{References}


1. Bakowska JC, and Morrell JI (1997). Atlas of the neurons that express mRNA for the long form of the prolactin receptor in the forebrain of the female rat. J Comp Neurol., 386: 161-177.

2. Bakowska JC, and Morrell JI (2003). The distribution of mRNA for the short form of the prolactin receptor in the forebrain of the female rat. Mol Brain Res. 116: 50-58.

3. Bronson FH, and Desjardins C (1982). Endocrine Responses to Sexual Arousal in Male Mice. Endocrinology [Internet].1982 Oct [cited 2017 Jul 18]; 111(4):1286-1291.

4. Brown RSE, Aoki M, Ladyman SR, et al. (2017). Prolactin action in the medial preoptic area is necessary for postpartum maternal nursing behavior. Proc Natl Acad Sci USA. 2017; 114:10779-10784.

5. Clement P, and Giuliano F. (2016). Physiology and pharmacology of ejaculation. Basic Clin Pharmacol Toxicol., 2016; 119:18-25.

6. Colao A, De Rosa M, Sarnacchiaro F et al. (1996). (Chronic treatment with CV 205-502 restore the gonadal function in hyperprolactinemic males. Eur J Endocrinol. 1996; 135: 548552.

7. Esteves, F., Matias, D., Mendes, A., Lacoste, B., and Lima, S. (2019). Sexually dimorphic neuronal inputs to the neuroendocrine dopaminergic system governing prolactin release. J Neuroendocrinol., 2019; 31: e12781.

8. Faulkner LD, Dowling AR, Stuart RC, Nillni EA, and Hill JW. (2015). Reduced melanocortin production causes sexual dysfunction in male mice with POMC neuronal insulin and leptin insensitivity. Endocrinol, 2015; 156(4):1372-1385.

9. Finkelstein JS, Lee H, Burnett-Bowie SAM, Pallais JC, Yu EW, Borges LF, Jones BF, Barry CV, Wulczyn KE, and Thomas BJ. (2013). Gonadal steroids and body composition, strength, and sexual function in men. New England J Med., 2013; 369: 10111022.

10. Gilbert DC, Duong T, Kynaston HG, Alhasso AA, Cafferty FH, Rosen SD, Kanaga-Sundaram S, Dixit S, Laniado M, and Madaan S. (2017). Quality-of-life outcomes from the Prostate Adenocarcinoma: TransCutaneous Hormones (PATCH) trial evaluating luteinising hormone-releasing hormone agonists versus transdermal oestradiol for androgen suppression in advanced prostate cancer. BJU Inter., 2017; 119: 667-675.

11. Guillaumot P, and Benahmed M. (1999). Prolactin receptors are expressed and hormonally regulated in rat Sertoli cells. Mol Cell Endocrinol. 1999; 149: 163-168.

12. Guillou A, Romanò N, Steyn F, Abitbol K, Le Tissier P, and Bonnefont X. (2015). Assessment of lactotroph axis functionality in mice: longitudinal monitoring of PRL secretion by ultrasensitive-ELISA. Endocrinol. 2015; 156(5): 1924-1930.

13. Hashemian, F., Shafigh, F., and Roohi, E. (2016). Regulatory role of prolactin in paternal behavior in male parents: Anarrative review. J Postgrad Med., 2016; 62(3): 182-187.

14. Kirk SE, Xie TY, Steyn FJ, Grattan DR, and Bunn SJ. (2017).Restraint stress increases prolactin-mediated phosphorylation of signal transducer and activator of transcription 5 in the hypothalamus and adrenal cortex in the male mouse. J Neuroendocrinol. 2017; 29(6).

15. Kokay IC, Wyatt A, Phillipps HR, Aoki M, Ectors F, Boehm U, et al. (2018). Analysis of prolactin receptor expression in the murine brain using a novel prolactin receptor reporter mouse. J Neuroendocrinol., 2018; 30(9): e12634.
16. McCabe MP, Sharlip ID, Lewis R, Atalla E, Balon R, Fisher $\mathrm{AD}$, Laumann E, Lee SW et al. (2016). Incidence and prevalence of sexual dysfunction in women and men: a consensus statement from the fourth international consultation on sexual medicine. J Sex Med., 2016; 13(2): 144-152.

17. Melis, M. R., and Argiolas, A. (2021). Oxytocin, Erectile Function and Sexual Behavior: Last Discoveries and Possible Advances. Inter J Mole Sci., 2021; 22(19): 10376.

18. Phan, J., Alhassen, L., Argelagos, A., Alhassen, W., Vachirakorntong, B., Lin, Z., Sanathara, N., and Alachkar, A. (2020). Mating and parenting experiences sculpture moodmodulating effects of oxytocin-MCH signaling. Scientific Reports. 2020; 10: 13611.

19. Rastrelli, G., Corona, G., and Maggi, M. (2015). The role of prolactin in andrology: what is new? Rev Endocr Metab Disord, 2015; 16: 233-248.

20. Raut, S., Deshpande, S., and Balasinor, NH. (2019).Unveiling the role of prolactin and its receptor in male reproduction. Horm Metab Res., 2019; 51: 215-219.

21. Rodrigues AR, Almeida H, and Gouveia AM. (2015). Intracellular signaling mechanisms of the melanocortin receptors: current state of the art. Cell Mol Life Sci., 2015; 72(7): 1331-1345.

22. Russell, N, and Grossmann, M. (2019). Mechanisms in endocrinology, Estradiol as a male hormone. European $\mathrm{J}$ Endocrinol. 2019; 181: R23-R43.

23. Salais-López, H., Agusti'n-Pavón, C., Lanuza, E., and Mart1'nez-Garc1'a, F. (2018). The maternal hormone in the male brain: Sexually dimorphic distribution of prolactin signalling in the mouse brain. PLoS ONE, 2018; 13(12): $\mathrm{e} 0208960$

24. Sanathara, N. M., Garau, C., Alachkar, A., Wang, L., Wang, Z., Nishimori, K., and Civelli, O. (2018). Melanin concentrating hormone modulates oxytocin-mediated marble burying. Neuropharmacol, 2018; 128: 22-32.

25. Schulster, M., Bernie, AM., and Ramasamy, R. (2016). The role of estradiol in male reproductive function. Asian $\mathrm{J}$ Androl, 2016; 18: 435-440.

26. Semple E, and Hill JW. (2018). Sim1 neurons are sufficient forMC4Rmediated sexual function in male mice. Endocrinol, 2018; 159(1): 439-449.

27. Semple, E., Shalabi, F., Hill, J. (2019). Oxytocin Neurons Enable Melanocortin Regulation of Male Sexual Function in Mice. Mole Neurobiol, 2019; 56: 6310-6323.

28. Stephen R. Hammes, Ellis R. and Levin. (2019). Impact of estrogens in males and androgens in females. J Clin Invest. 2019; 129(5): 1818-1826.

29. Wibowo E and Wassersug RJ. (2013). The effect of estrogen on the sexual interest of castrated males: implications to prostate cancer patients on androgen-deprivation therapy. Crit Rev Oncol/Hematol., 2013; 87, 224-238.

30. Yao, Y., Fu, L. Y., Zhang, X. and van den Pol, A. N. (2012). Vasopressin and oxytocin excite $\mathrm{MCH}$ neurons, but not other lateral hypothalamic GABA neurons. Am. J. Physiol. Regul. Integr. Comp. Physiol., 2012; 302(7): R815-824. 